\title{
A Case of Sinonasal Extranodal NK/T-Cell Lymphoma with Indolent Behaviour and Low-Grade Morphology
}

\author{
Valentina Tabanelli' ${ }^{1}$, Riccardo Valli², Magda Zanelli², Simona Righi', Anna Gazzola1, \\ Claudia Mannu${ }^{1}$, Stefano Pileri ${ }^{1}$, Elena Sabattini ${ }^{1}$ \\ ${ }^{1}$ Hematopathology and Haematology Sections, Department of Experimental, Diagnostic and Specialty \\ Medicine, S. Orsola-Malpighi Hospital, University of Bologna, Bologna, Italy \\ ${ }^{2}$ Anatomic Pathology Unit, IRCSS Santa Maria Nuova Hospital, Reggio Emilia, Italy \\ Email: valentina.tabanelli@gmail.com
}

Received 11 September 2014; revised 10 October 2014; accepted 9 November 2014

Copyright (C) 2014 by authors and Scientific Research Publishing Inc.

This work is licensed under the Creative Commons Attribution International License (CC BY).

http://creativecommons.org/licenses/by/4.0/

c) (i) Open Access

\begin{abstract}
Extranodal NK/T-cell lymphoma, nasal type (ENKL), is an uncommon subtype of EBV associated lymphoma usually characterized by aggressive clinical course. We report an atypical sinonasal ENKL case with long-lasting indolent behaviour, developed in the setting of a polymorphic EBVassociated lymphoproliferative disorder (LPD). A 52-year-old woman had suffered from chronic sinusitis and nasal obstruction since 2000, moderately worsened during the last years (marked enlargement of the sino-nasal mucosa at MRI in 2011) with elevated anti-VCA IgG and IgM titers. Three subsequent biopsies revealed slightly increasing morphophenotypic atypia, ranging from a polymorphic B- and T-cell EBV positive proliferation (diagnosed in 2011, but not fulfilling CAEBV diagnostic criteria) to an overt monomorphic mildly atypical T LPD without necrosis and angiocentricty diagnosed as ENKL in 2013 upon immunophenotype and TCR- $\gamma$ gene clonal rearrangement. Clinically indolent ENKL with low-grade morphology is extremely rare and diagnostically challenging; while the few reports in the literature describe long-survival in ENKL treated patients comparing histologically neoplastic lesions at onset and recurrences, no reports are published on the slow progression from a polymorphic EBV-related T/NK proliferation to a histologically overt clinically indolent ENKL in an untreated patient who only received occasional steroid administration.
\end{abstract}

\section{Keywords}

ENKL, Indolent, CAEBV, EBV

How to cite this paper: Tabanelli, V., Valli, R., Zanelli, M., Righi, S., Gazzola, A., Mannu, C., Pileri, S. and Sabattini, E. (2014) A Case of Sinonasal Extranodal NK/T-Cell Lymphoma with Indolent Behaviour and Low-Grade Morphology. Case Reports in Clinical Medicine, 3, 596-600. http://dx.doi.org/10.4236/crcm.2014.311129 


\section{Introduction}

Extranodal natural killer (NK)/T-cell lymphoma, nasal type (ENKL), is an uncommon subtype of lymphoma derived from NK or, less commonly, from cytotoxic T-cells, associated with Epstein-Barr virus (EBV), which usually arises in the upper aerodigestive tract [1]. It displays a peculiar geographical distribution, being extremely uncommon in Western countries with a predilection for Asian and South American populations; it is considered an aggressive form of non-Hodgkin lymphoma with a poor prognosis [1]. ENKL encompasses a morphological broad spectrum, varying from cases with angiocentric atypical cell infiltrate with extensive necrosis to small cell variants simulating an inflammatory process [1] [2]. We report an atypical case of sinonasal NK/T-cell lymphoma with indolent biological behaviour, developed in the setting of a polymorphic EBV-associated lymphoproliferative disorder.

\section{Case Report}

A 52-year-old woman with a history of interferon-treated HCV-related chronic hepatitis and healed HBV infection, had suffered from chronic sinusitis and nasal obstruction since 2000, with periodic exacerbations for which she received steroids and antibiotics.

Obstruction symptoms and radiological findings had progressively worsened during the last five years and a MRI performed in 2011 showed marked thickening of the sino-nasal mucosa, obliterating the right maxillary sinus and the nasal lower turbinate. Laboratory tests for EBV showed elevated anti-viral capsid antigen (VCA) IgG and IgM titers and low baseline plasma EBV DNA levels. Immune system abnormalities were ruled out; HIV serology was negative.

A biopsy of the nasal mucosa made in 2011 revealed a mixed B/T lymphoid population, admixed with polyclonal plasma cells, without neither cytological atypia nor necrosis. EBV encoded RNA by in situ hybridization (EBER) was largely positive; CD3+ lymphocytes regularly expressed the other T-cell antigens (CD2, CD5, CD7) and CD56 was negative: therefore, a diagnosis of reactive polymorphic EBV-associated lymphoid proliferation was made (Figure 1).

On February 2013, respiratory symptoms worsened and fever and night sweating developed; the anti-VCA antibodies were still increased and the sino-nasal lesion was still evident at CT in absence of organomegalies and lymphadenopathies. On July 2013 a second biopsy was performed: rhino-pharyngeal mucosa was superficially infiltrated by a prevalent T-cell population of small to medium-sized cells with mildly irregular nuclei that

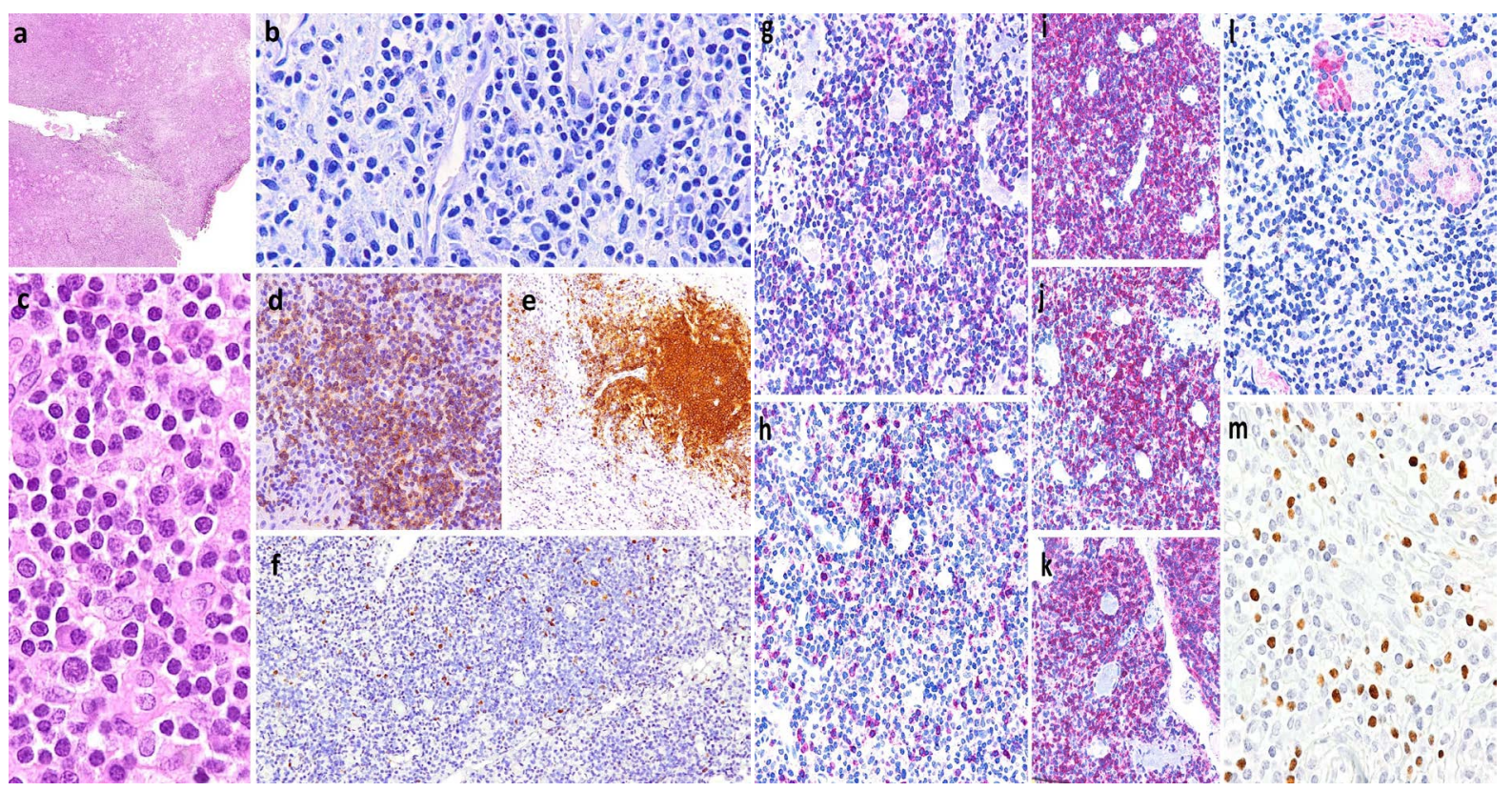

Figure 1. First biopsy of the nasal mucosa (a); Giemsa (40×) (b); H\&E (40×) (c); CD3 (d); CD20 (e); Ki67 (f); CD4 (g); CD8 (h); CD2 (i); CD5 (j); CD7 (k); CD56 (l); EBER (m). 
showed no angioinvasiveness nor necrosis, but a superficial mucosal ulceration. A defective phenotype could be detected with positive CD3, CD2, CD56 and cytotoxic markers (TIA-1, Granzyme-B) but negative CD4, CD8, TCR-Beta F1 and TCR- $\gamma / \delta$ molecules and aberrant loss of CD5 and CD7. The Ki67 index was moderately high. As previously observed EBER probe was diffusely positive (Figure 2).

Given the persistence of the nasal mass a third biopsy was performed three months later, exhibiting the same histological features described in the previous one. Polymerase chain reaction (PCR) analyses of the T-cell receptor genes were performed, revealing an emerging clonal rearrangement of the TCR- $\gamma$ gene in a polyclonal setting (Figure 3). Thus, diagnosis of NK/T-cell lymphoma, nasal type was finally made.
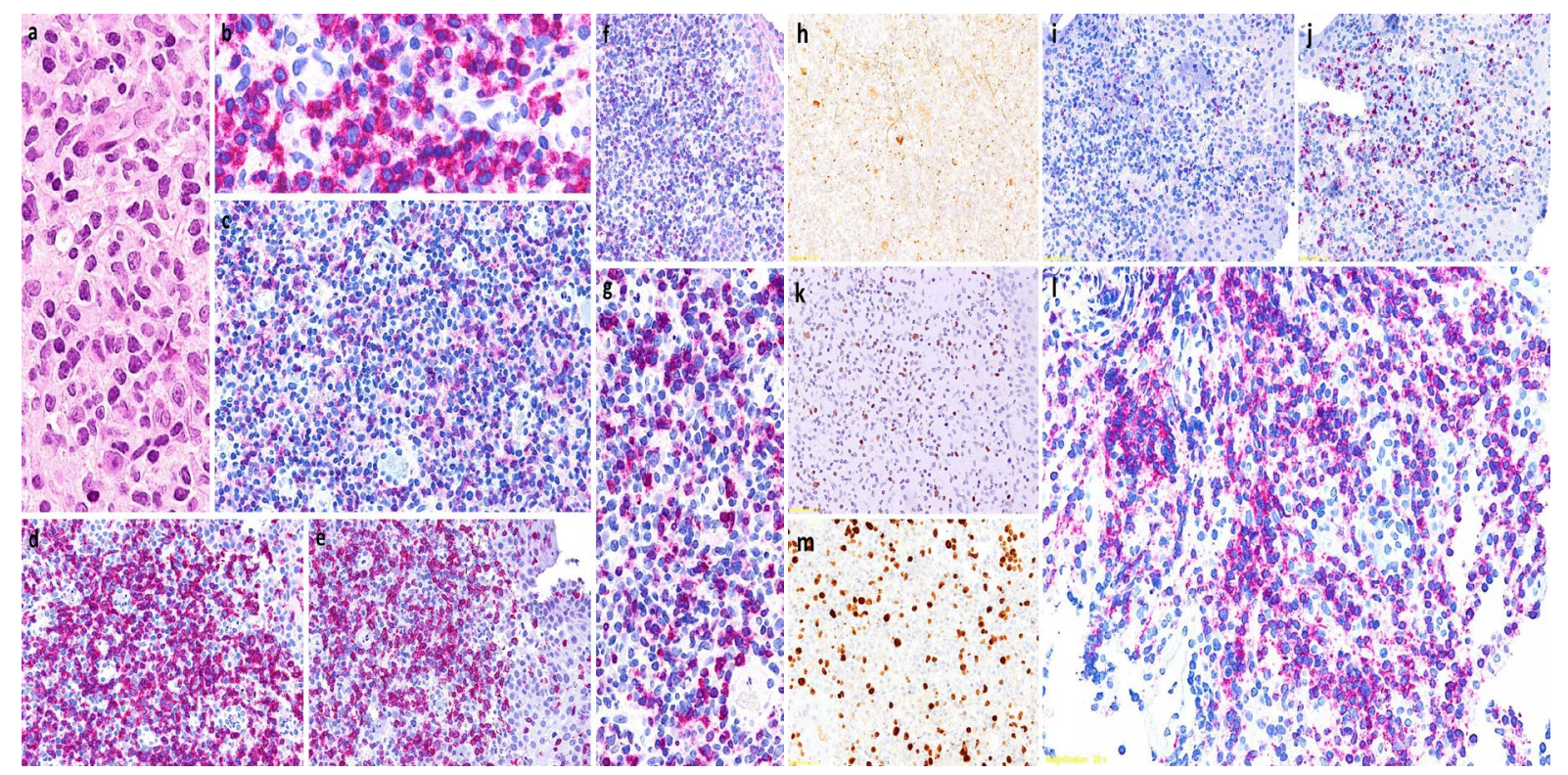

Figure 2. Second biopsy of the nasal mucosa: H\&E (40×) (a); CD3 (b); CD4 (c); CD2 (d); CD5 (e); CD8 (f); CD7 (g); Granzyme B (h); perforin (i); Tia-1 (j); EBER (k); CD56 (l); Ki67 (m).
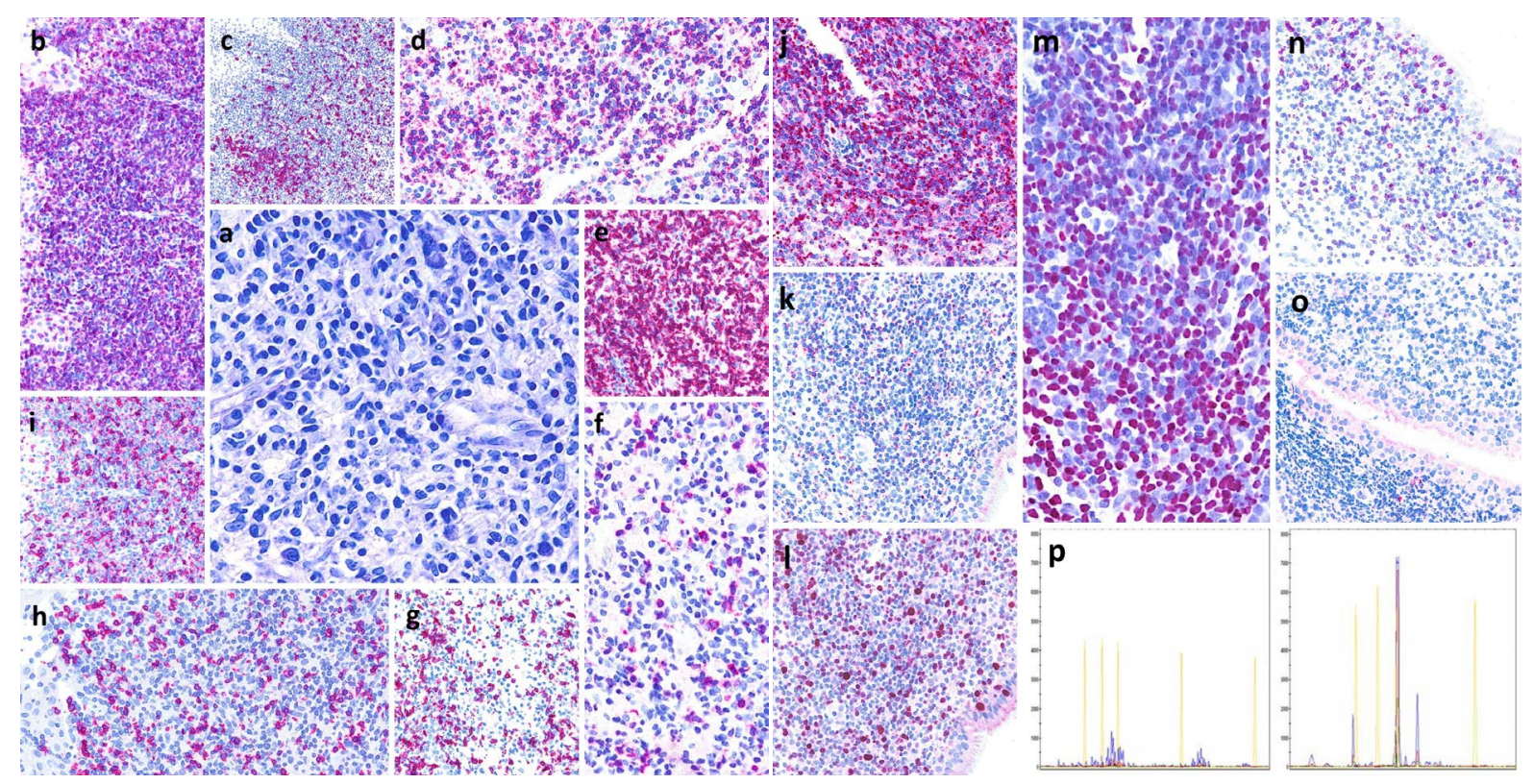

Figure 3. Third biopsy of the nasal mucosa: Giemsa (40×) (a); CD3 (b); CD20 (c); CD56 (d); CD2 (e); CD5 (f); CD7 (g); CD4 (h); CD8 (i); Tia-1 (j); Granzyme B (k); KI67 (l); EBER (m); TCR-Beta F1 (n); TCR $\gamma / \delta(o)$; PCR analysis: clonal rearrangement of the TCR $\gamma$ gene $(\mathrm{p})$. 


\section{Discussion}

ENKL is an aggressive disease whose prognosis is generally unfavourable and has been related to several factors, such as extranasal site of involvement, stage, performance status, EBV-DNA levels and treatment [3]-[6].

The first notable feature of our case is the long lasting indolent biological behavior. Slightly better survival rate were actually already reported in primary cutaneous lesions [7] where two cases run a remarkably favourable course of 10 and 22 years [8] [9]. Considering the nasal area, two recent series described ENKL cases with late relapses (ranging from 40 months to 10 - 29 years) after chemotherapy induced remission, among which 2 and 8 patients respectively showed nasal presentation [10] [11]; an additional report dealt with an exceptional case of recurrence after 38 years remission [12]. However in all the aforementioned cases the long lasting behavior was induced by treatment response since they were all treated with radiotherapy and/or chemoradiotherapy: conversely, to the best of our knowledge, our patient is the first described with a slow disease progression from an EBV positive polymorphic B/T-cell mixed infiltrate to a more monomorphic T-cell one in the absence of any aggressive treatment since she received only steroids.

The morpho-phenotypical differences between the first and the last two biopsies deserve a comment: what observed in 2011 resembled the category A1 of an EBV-associated T/NK-cell lymphoproliferative disorder (EBV-T/NK LPD) as described by Oshima et al. [13], who defined this category as polymorphic. Since nasal obstruction related symptoms had been present, though at varying degrees, for 13 years before the recent worsening, it is likely that this type of mixed polymorphic infiltrate had persisted for such a long period before giving transforming into a more monomorphic T/NK cell disorder. Many EBV-T/NK LPD cases clinically overlap with chronic active EBV infection which usually develops in children and young adults, although it can be rarely detected in middle-aged and elderly; CAEBV diagnostic criteria are largely clinical and are represented by high viral load in peripheral blood or tissues, recurrent or persistent infectious mononucleosis-like symptoms for at least 3 months and absence of immunodeficiency [1]. In CAEBV setting, polyclonal EBV+NK/T-cells may evolve to a monoclonal proliferation giving rise to a frank lymphoid malignancy. Although we cannot exclude that our lesion developed in the context of a CAEBV (presence of fever and high anti-VCA titers) the diagnostic criteria were not completely fulfilled (no lymphadenopathies, no hepatosplenomegaly, no mosquito allergy; no typical sore-throat episodes). Nonetheless, as observed in EBV-T/NK LPD and/or CAEBV a non-malignant EBV-driven small NK-cell clone may have been selected with time and expanded between the first and second/ third biopsy giving rise to a more frank T cell lymphoma.

However, the latter was a challenging diagnosis which was mainly made upon the aberrant immunophenotype given the low-grade histological appearance of the process, with no evidence of angiocentric growth or necrosis. Hasserjian et al. [2] reported 3 similar cases (one of them with a 10-year long history), composed by small lymphocytes lacking striking atypia and mimicking atypical inflammatory sino-nasal infiltrates.

\section{Conclusion}

In conclusion ENKL with indolent course and low-grade morphology is extremely rare and represents a diagnostic challenge. The case herein described represents the first one seen at onset arising from a pre-existing polymorphic EBV-related T/NK proliferation.

\section{References}

[1] Swerdlow, S.H., Campo, E., Harris, N.L., Jaffe, E.S., Pileri, S.A., Stein, H., et al., Eds. (2008) WHO Classification of Tumours of Haematopoietic and Lymphoid Tissues. IARC Press, Lyon.

[2] Hasserjian, R.P. and Harris, N.L. (2007) A Spectrum of Tumors with Variable Manifestations and Immunophenotype. American Journal of Clinical Pathology, 127, 860-868. http://dx.doi.org/10.1309/2F39NX1AL3L54WU8

[3] Chim, C.S., Ma, S.Y., Au, W.Y., Choy, C., Lie, A.K.W., Liang, R., et al. (2004) Primary Nasal Natural Killer Cell Lymphoma: Long-Term Treatment Outcome and Relationship with the International Prognostic Index. Blood, 103, 216-221. http://dx.doi.org/10.1182/blood-2003-05-1401

[4] Suzuki, R., Suzumiya, J., Yamaguchi, M., et al. (2010) Prognostic Factors for Mature Natural Killer (NK) Cell Neoplasms: Aggressive NK Cell Leukemia and Extranodal NK Cell Lymphoma, Nasal Type. Annals of Oncology, 21, 10321040. http://dx.doi.org/10.1093/annonc/mdp418

[5] Huang, H., Lin, Z., Lin, X., et al. (2011) Long-Term Outcomes of Patients with Newly Diagnosed Extranodal Natural Killer/T-Cell Lymphoma Treated by Etoposide, Prednisone, Vincristine, Cyclophosphamide, and Doxorubicin Regi- 
men: A Single-Institution Experience. Leuk Lymphoma, 52, 1041-1048. http://dx.doi.org/10.3109/10428194.2011.561388

[6] Hsieh, P.P., Tung, C.L., Chan, A.B.W., et al. (2007) EBV Viral Load in Tumour Tissue Is an Important Prognostic Indicator for Nasal NK/T-Cell Lymphoma. American Journal of Clinical Pathology, 128, 579-584. http://dx.doi.org/10.1309/MN4Y8HLQWKD9NB5E

[7] Choi, Y.L., Park, J.H., Namkung, J.H., et al. (2009) Extranodal NK/T Cell Lymphoma with Cutaneous Involvement: “Nasal” vs. "Nasal-Type” Subgroups: A Retrospective Study of 18 Patients. British Journal of Dermatology, 160, 333337. http://dx.doi.org/10.1111/j.1365-2133.2008.08922.

[8] Watabe, D., Kanno, H., Inoue-Narita, T., Onodera, H., Izumida, W., Kowata, S., et al. (2009) A Case Of Primary Cutaneous Natural Killer/T-Cell Lymphoma, Nasal Type, With Indolent Clinical Course: Monoclonal Expansion Of Epstein-Barr Virus Genome Correlating with the Terminal Aggressive Behaviour. British Journal of Dermatology, 160, 205-207. http://dx.doi.org/10.1111/j.1365-2133.2008.08892.x

[9] Zuriel, D., Fink-Puches, R. and Cerroni, L. (2012) A Case of Primary Cutaneous Extranodal Natural Killer/T-Cell Lymphoma, Nasal Type, with a 22-Year Indolent Clinical Course. The American Journal of Dermatopathology, 34, 194-197. http://dx.doi.org/10.1097/DAD.0b013e318230ee41

[10] Kim, S.J., Park, Y., Kim, B.S., Kim, I., Ko, Y.H., Kim, W.S., et al. (2012) Extranodal Natural Killer/T-Cell Lymphoma with Long-Term Survival and Repeated Relapses: Does It Indicate the Presence of Indolent Subtype? The Korean Journal of Hematology, 47, 202-206. http://dx.doi.org/10.5045/kjh.2012.47.3.202Au

[11] Au, W.Y., Kim, S.J., Yiu, H.H., Ngan, R.K., Loong, F., Kim, W.S., et al. (2010) Clinicopathological Features and Outcome of Late Relapses of Natural Killer Cell Lymphomas 10-29 Years after Initial Remission. American Journal of Hematology, 85, 362-363. http://dx.doi.org/10.1002/ajh.21663.

[12] Schrader, C., Janssen, D., Kneba, M. and Lennert, K. (2004) A 38-Year History of Natural-Killer-Cell Lymphoma. The New England Journal of Medicine, 350, 418-419. http://dx.doi.org/10.1056/NEJM200401223500423

[13] Ohshima, K., Kimura, H., Yoshino, T., Kim, C.W., Ko, Y.H., Lee, S.S., et al., CAEBV Study Group (2008) Proposed Categorization of Pathological States of EBV-Associated T/Natural Killer-Cell Lymphoproliferative Disorder (LPD) in Children and Young Adults: Overlap with Chronic Active EBV Infection and Infantile Fulminant EBV T-LPD. Pathology International, 58, 209-217. http://dx.doi.org/10.1111/j.1440-1827.2008.02213.x 
Scientific Research Publishing (SCIRP) is one of the largest Open Access journal publishers. It is currently publishing more than 200 open access, online, peer-reviewed journals covering a wide range of academic disciplines. SCIRP serves the worldwide academic communities and contributes to the progress and application of science with its publication.

Other selected journals from SCIRP are listed as below. Submit your manuscript to us via either submit@scirp.org or Online Submission Portal.
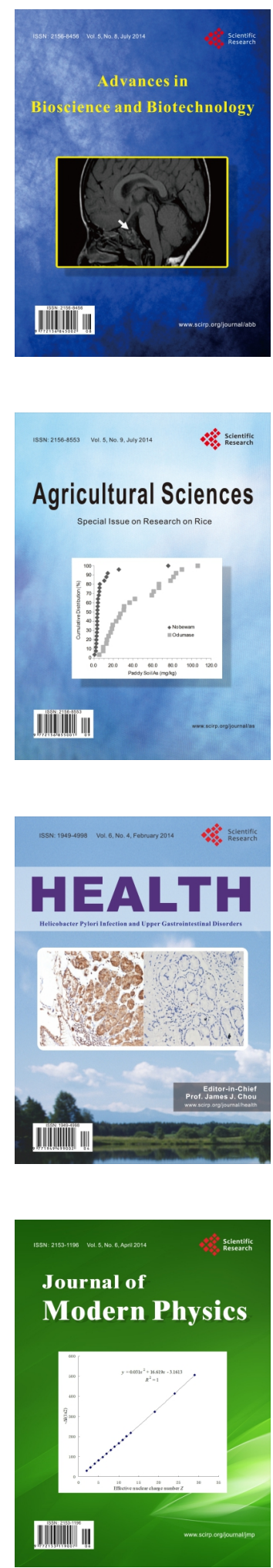
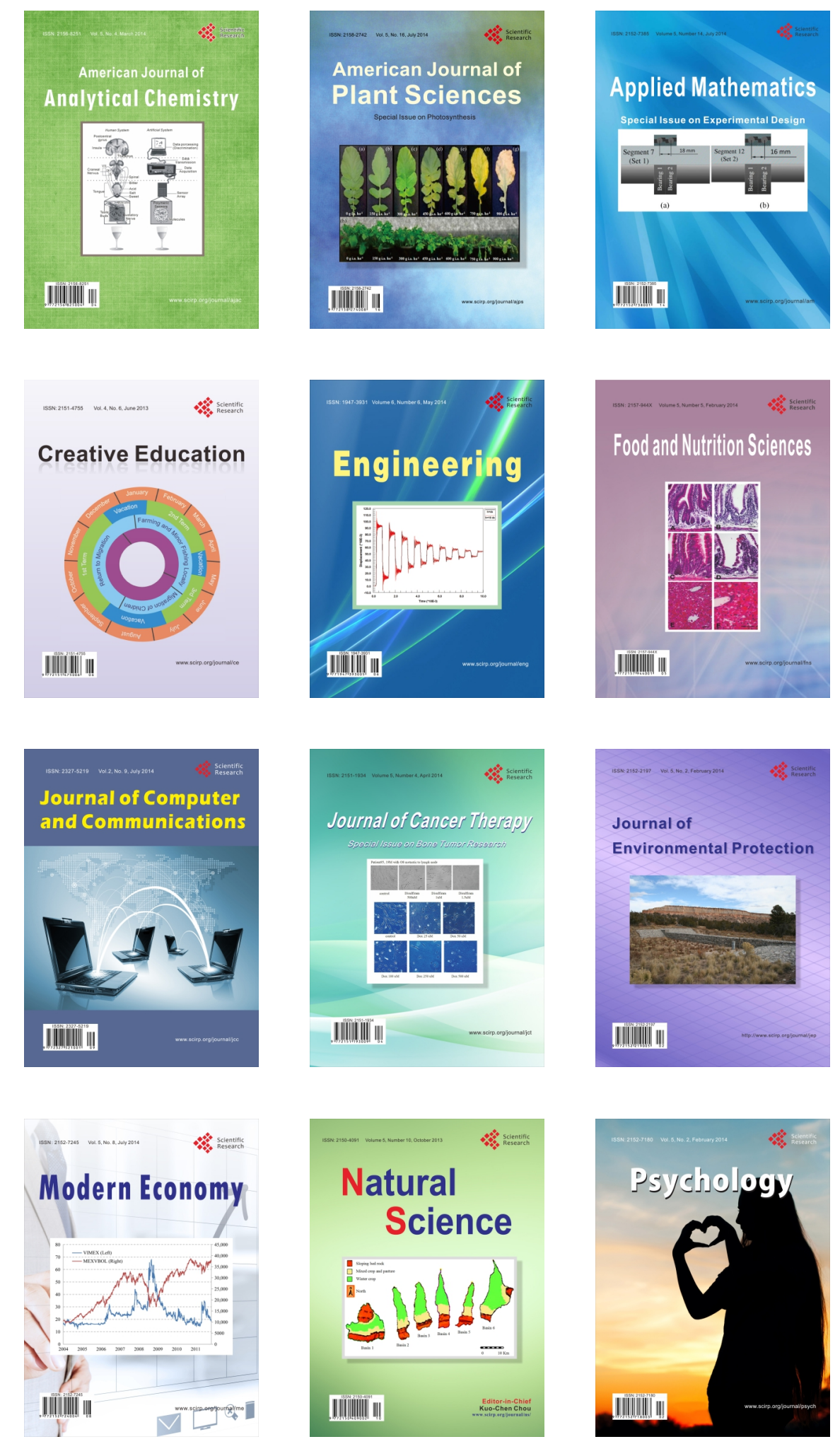